\title{
Electrical model and optimal design scheme for low work-function tethers in thrust mode
}

\author{
G. Sánchez-Arriagd ${ }^{a, *}$, J.R. Sanmartín ${ }^{\mathrm{b}}$ \\ a Bioengineering and Aerospace Engineering Departnent, Universidad Carios II de Madrid, Leganés, Madrid, 28911, Spain \\ b Departanento de F́sica Aplicada a las ingenierías Aeronáutica y Naval, Escuela Técnica Superior de Ingenieria Aeronáutica y del Espacio. \\ Universidad Politécnica de Madrid, Plaza Cardenal Cisneros 3, 28040 Madrid, Spain
}

\section{A R T I C L E I N F O}

\section{Article history:}

Received 12 February 2019

Received in revised form 8 october 2019

Accepted 25 October 2019

Available online 30 October 2019

\begin{abstract}
A B S T R A C T
A low work-function tether (LWT), a subclass of bare electrodynamic tether made of a conductor partially coated with a low work function material, can exchange momentum and energy with planetary magnetospheres without any consumable. If fed by an onboard power source to reverse the natural direction of the current given by the motional electric field, a LWT can produce an useful thrust in drag compensation and re-boost scenarios. In the considered scheme, the LWT has an anodic bare segment for passive electron collection, followed by an insulated segment, a power source, and a cathodic segment that emits electrons passively through thermionic and photoelectric effects. Current and voltage profiles along the LWT are obtained and used to compute the system efficiency, i.e. the electrical power to mechanical power conversion rate, and the minimum electrical power to avoid space-charge effects in the cathodic segment. The design conditions to reach a high-efficiency regime are presented. For a given orbit and required thrust, optimal values for the tether geometry. including the fractional lengths of all three tether segments, are found. The results are applied to the preliminary design of a LWT system that would compensate the aerodynamic drag on the International Space Station. The analysis shows that the mission can be performed by a LWT fed with a power source of $4.7 \mathrm{~kW}$. length, width and thickness equal to $5.1 \mathrm{~km}, 2 \mathrm{~cm}$ and $30 \mu \mathrm{m}$. work function $1.5 \mathrm{eV}$, and temperature $6.00 \mathrm{~K}$.
\end{abstract}

S 2019 Elsevier Masson SAS. All rights reserved.

\section{Introduction}

The search of alternatives to conventional propulsion technologies that could lower the cost of space operations is a matter of high interest for the sector. Moreover, thorough analysis [1] indicates that the space debris population in Low Earth Orbit (LEO) is under the Kessler syndrome [2] and an increasing interest exists on developing new technologies for deorbiting and re-boosting spacecraft. Since the propellant represents an important fraction of mass in conventional devices, propellant-less technologies working under physical principles different to the action-reaction law are under investigation. One of them is the sail, a drag augmentation device that uses the aerodynamic drag. However, a sail both produces no re-boost and deorbits inefficiently from the altitudes of interest for space debris due to the very low air density. On the other hand, electrodynamic tethers, i.e. long conductors that exchange momentum with the Earth's magnetosphere, have been discussed broadly as an interesting alternative [3-8]. The peak on tether interest reached at the end of the last century, when many missions were flown, was followed by a period with important theoretical progresses on key aspects like tether survivability [9-11], mission design [12], dynamics [13] and control [14,15], among others. Two tether missions, DESCENT [16] and TEPCE that are planned for 2019, and very recent works on a great variety of topics, such as tether formation systems [17], tethered space tug-systems [18], tether-assisted payload return with length control [19], show that space tethers are nowadays an active field.

The standard electrodynamic tether concept till 2012 was a bare conductor as passive electron collector equipped with an active electron emitter [20]. Such a system is propellant-free, but it involves an active element that, in the case of a Hollow Cathode (HC), needs expellant. An important progress towards simplicity was given when the themionic tether was introduced in 2012 [21]. Instead of carrying an active electron emitter, the tether is coated with a low work-function (W) material that emits electron passively through the themionic effect, thus yielding a system totally free of consumable. Few years later, it was pointed out that the photoelectric effect can be also an important emission mechanism for tether coated with low- $W$ materials and the term low Work-Function Tether (LWT), which includes thermionic and pho- 


\section{Nomenclature}

$A_{t} \quad$ tether cross-sectional area, $\ldots \ldots \ldots \ldots \ldots \ldots \ldots, \mathrm{m}^{2}$

B ambient magnetic field, $\ldots \ldots \ldots \ldots \ldots \ldots \ldots \ldots \ldots, \mathrm{T}$

$c_{c h} \quad$ jet speed of chemical thruster, $\ldots \ldots \ldots \ldots \ldots \ldots . \ldots \mathrm{m} / \mathrm{s}$

$c_{e} \quad$ jet speed of electrical thruster,$\ldots \ldots \ldots \ldots \ldots \ldots . \mathrm{m} / \mathrm{s}$

$E_{m} \quad$ motional electric field component along $\mathbf{u}_{\mathbf{t}}, \ldots \ldots \mathrm{V} / \mathrm{m}$

$E$ photon energy, ............................... eV

$\varepsilon \quad$ elementary charge, $\ldots \ldots \ldots \ldots \ldots \ldots \ldots \ldots \ldots \ldots, C$

$\mathbf{F}_{0} \quad$ aerodynamic drag, $\ldots \ldots \ldots \ldots \ldots \ldots \ldots \ldots \ldots \ldots, \mathrm{N}$

$\mathbf{F}_{M} \quad$ Lorentz force, $\ldots \ldots \ldots \ldots \ldots \ldots \ldots \ldots \ldots \ldots \ldots, \ldots$

$h_{\mathrm{t}}$ tether thickness (or tadius), ................ m

I tether current, ............................. A

$J_{a v} \quad$ average current, $\ldots \ldots \ldots \ldots \ldots \ldots \ldots \ldots \ldots \ldots \ldots \ldots$ A

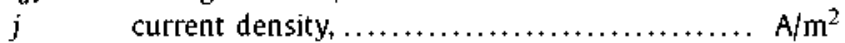

$k_{B} \quad$ Boltzmann constant, ................... $\mathrm{m}^{2} \mathrm{~kg} / \mathrm{s}^{2} \mathrm{~K}$

$\mathrm{L} \quad$ tether length, $\ldots \ldots \ldots \ldots \ldots \ldots \ldots \ldots \ldots \ldots \ldots \ldots, \mathrm{m}$

$L_{a} \quad$ anodic segment length, $\ldots \ldots \ldots \ldots \ldots \ldots \ldots \ldots \ldots, \ldots$

$L_{i} \quad$ cathodic segment length $, \ldots \ldots \ldots \ldots \ldots \ldots \ldots \ldots, m$

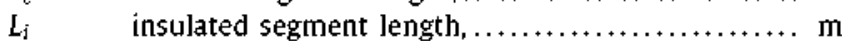

$L^{*} \quad$ length characterizing ohmic effects $, \ldots \ldots \ldots \ldots \ldots, m$

$M_{d} \quad$ system mass, $\ldots \ldots \ldots, \ldots \ldots \ldots, \ldots \ldots \ldots, \ldots, \ldots, k g$

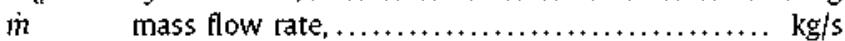

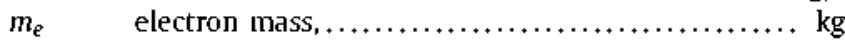

$m_{t} \quad$ tether mass, $\ldots \ldots \ldots, \ldots, \ldots, \ldots, \ldots, \ldots, \ldots, \ldots, \mathrm{kg}$
$N_{0}$

$p_{t}$

$S$

$T_{e}$

$T_{i}$

$T_{t}$

$\mathbf{u}_{t}$

v

$W$

$W_{E}$

$W_{M}$

$w_{t}$

$x$

$Y_{p h}$

$\alpha_{t}$

$\alpha$

$\eta_{e}$

$\eta_{e f f}$

$\Phi$

$\rho_{t}$

$\sigma_{t}$

$\tau$ ambient plasma density, ................. $1 / \mathrm{m}^{3}$

tether perimeter, ..............................m m

Solar energy spectrum, ................. $\mathrm{ph} / \mathrm{sm}^{2} \mathrm{eV}$

electron temperature, ........................ K

ion temperature $, \ldots \ldots \ldots \ldots \ldots \ldots \ldots \ldots \ldots \ldots \ldots, \mathrm{K}$

tether temperature, $\ldots \ldots \ldots \ldots \ldots \ldots \ldots \ldots \ldots \ldots \ldots, \mathrm{K}$

unit vector along the straight tether

spacecraft velocity,......................... $\mathrm{m} / \mathrm{s}$

work function, $\ldots \ldots \ldots \ldots \ldots \ldots \ldots \ldots \ldots \ldots, \ldots, \mathrm{eV}$

electrical power, ............................W

mechanical power, .......................... W

tether width $, \ldots \ldots \ldots \ldots \ldots \ldots \ldots \ldots \ldots \ldots \ldots \ldots, \ldots, \ldots$

distance along the tether, .................... m

tether photoelectron yield,.................. el/ph

tether related hardware factor

inverse specific power,..................... kg/kw

thruster conversion efficiency

tether efficiency

local bias, ................................. $\mathrm{V}$

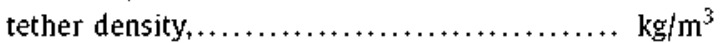

tether conductivity, ..................... 1/ $/ \mathrm{m}$

thruster allotted time $, \ldots \ldots \ldots \ldots \ldots \ldots \ldots \ldots \ldots, \ldots$ toelectric tethers, was proposed [22]. Besides deorbiting [21], system arrangements based on LWTs for power generation [23] and thrust production [24] have been discussed.

In parallel with development activities on LWT manufacturing, theoretical analyses are necessary to construct an envelope with tether performance and requirements, such as work function and temperature. However, except a work on deorbiting from Geostationary Transfer Orbit [25], the mission analysis of LWTs in space applications is an unspoiled field of research. Among the rich range of potential applications, this work studies for the first time thrust missions by [WTs. In general, tethers are certainly a suitable technology for very long thrust operations $[26,27]$. These missions include protracted drag compensation or repeated use to move payloads to higher orbits, as space 'tugs', freely re-boosting to meet, dock and grapple a new payload. A drag compensation scenario of particular interest is the re-boost of the International Space Station [28-30]. A $10 \mathrm{~km}$ long bare tether using less than $10 \mathrm{~kW}$ of power was proposed for ISS drag makeup, in the 1990's, soon after the bare-tether concept was introduced in 1993. Estimations suggested that 66 (out of 78 ) tons of propellant could be saved over a 10-year lifetime, at maximum duty cycle. Tether dynamics and thrusting raised concerns, however, about impact on microgravity payloads and safety issues, at Shuttle rendezvous and flight path [28].

The purpose of this work is to present an electrical model for IWTs in thrust mode and find optimal tether design conditions. Some relevant applications of the study include the re-boost of the ISS and also other extremely large, long-time operative satellites in LEO. New Space Stations might take the place of doomed ones, like the Russian MIR or the Chinese Tiangong-1, re-entered on 23 March 2001 and 2 April 2018, respectively. After revisiting in Sec. 2 some general considerations and key figures of merit about inspace propulsion systems, this work presents the following results. Section 3 introduces an electrical model for a LWT in thrust mode. The model is used to write the efficiency of the LWT, measured in terms of the effectiveness to transform onboard electric power into useful mechanical power, as a function of the design parameters like the lengths of the different tether segments. The design conditions to optimize the efficiency of the LWT are investigated analytically for the long tether limit in Sec. 4.1, and numerically for a general case in Sec. 4.2. The results are discussed and applied to the re-boost of the ISS in Sec. 5. Section 6 summarizes the conclusions.

\section{Figures of merit of space thrusters}

In comparing thrusting alternatives, the figure of merit, which must be low for high efficiency, is the thrusting-system inverse specific impulse, which is the ratio $M_{t} / F \tau$ between dedicated system mass $M_{t}$ and mission impulse provided by an average thrust $F$ during an allotted time $\tau$. For electric propulsion, $M_{t}$ includes propellant consumed at a mass-flow-rate $\dot{m}$ and power plant $\alpha W_{E}$, where $\alpha$ is the inverse specific power of supply and $W_{E}$ the electrical power [26],

$\frac{M_{d}}{F \tau}=\frac{\dot{m} \tau+\alpha W_{E}}{\dot{m}^{\prime} c_{e} \tau}=\frac{1}{c_{e}}+\frac{\alpha c_{e}}{2 \eta_{e} \tau}$

where $\eta_{e} \equiv F c_{e} / 2 W_{E}$ and $F=\dot{m} c_{e}$. With reference values of jet speeds $c_{e} \sim 30 \mathrm{~km} / \mathrm{s}$ and $15 \mathrm{~km} / \mathrm{s}$, overall conversion efficiencies $\eta_{e} \sim 0.65$ and 0.50 are found for lon and Hall thrusters, respectively. Also, $\alpha$ is typically a few tens of $\mathrm{kg} / \mathrm{kW}$ if a dedicated solar-plant is required, as for a space tug, and just several $\mathrm{kg} / \mathrm{kW}$ otherwise, as in the case of the Intemational Space Station.

In a so-called thruster mode, a tether also requires a power supply lying at the top of a tether collecting electrons, at the satellite, to invert anodic and cathodic ends by overcoming the electromotive force of the magnetically induced motional field $\boldsymbol{v}_{r e l} \times \boldsymbol{B}$, thus inverting current direction (electrons flowing upwards for eastward LEO orbits). Here $\boldsymbol{v}_{\text {tet }}$ is the tether-to-plasma relative velocity and $\boldsymbol{B}$ the ambient magnetic field. In the case of a standard tether with active electron emitter, electrons collected in a lower bare segment of length $L$ would be re-emitted in top cathodicexchange above the power supply, through a $\mathrm{HC}$ or a thermionic emitter. In the actual LWT case of interest, thermionic and photoelectric emissions along an upper tether segment coated with a low-W material replace the HC [24] (see Fig. 1). 


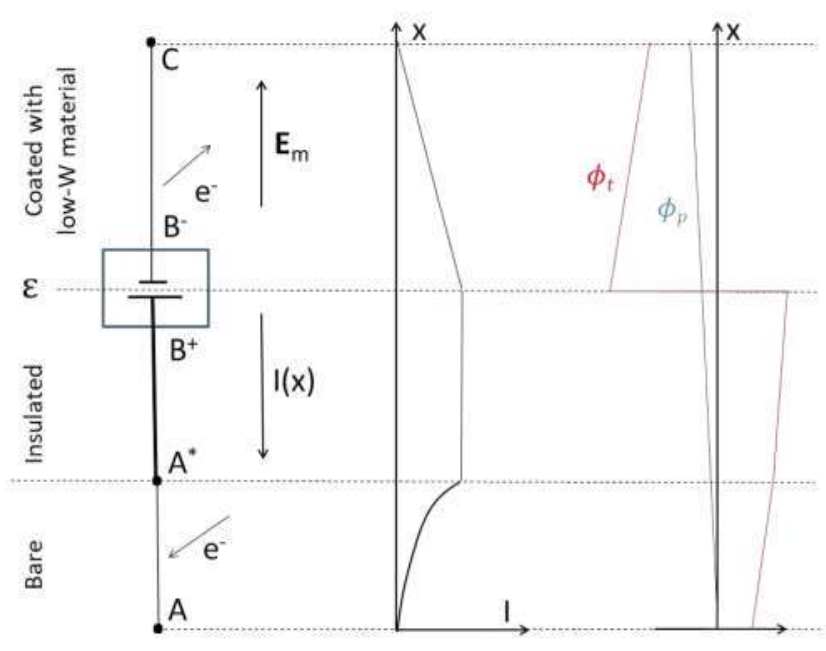

Fig. 1. Tether configuration in thrust mode.

A LWT involves no consumable mass; on the other hand tether mass $m_{t}$, times a factor $\alpha_{t} \sim 2-2.5$, accounting for directly related hardware, does contribute substantially to system mass $M_{d}$, thus written as $\alpha W_{E}+\alpha_{t} m_{t}$. In the HC case, its hardware, but not the expellant for typically not too long missions, makes an accountable contribution to mass $M_{d}$. Considering the tether as part of the power plant, we may write

$$
\frac{M_{d}}{F \tau} \simeq \frac{\alpha W_{E}+\alpha_{t} m_{t}}{\boldsymbol{F}_{M} \tau} \approx \frac{\alpha v}{\tau} \frac{W_{E}}{W_{M}}\left(1+\frac{\alpha_{t} m_{t}}{\alpha W_{E}}\right) \equiv \frac{\alpha v}{\tau \eta_{\text {eff }}}
$$

where $W_{M}=\boldsymbol{F}_{M} \cdot \boldsymbol{v}$ and $\boldsymbol{F}_{M}$ are the mechanical power and Lorentz force, $\boldsymbol{v}$ the orbital velocity, and $\eta_{\text {eff }}$ is an overall conversion efficiency, which might typically be about $1 / 3$ if using a HC [26].

Electric propulsion may be considered somewhat midway, in the route to make less and less use of consumables. On one extreme one finds rocket propulsion, which involves just consumable mass and has inverse specific impulse

$\frac{M_{d}}{F \tau}=\frac{1}{c_{c h}}$,

with $c_{c h}$ the chemical jet speed. The other extreme is tether propulsion (see Eq. (2)), which has no consumable mass (LWT) or small amount of expellant (bare tether with HC) but involves tether and associated hardware mass. Comparing the right-handsides of Eqs. (1)-(3), shows how this route leads to higher and higher specific impulse. With $c_{c h}$ definitely below $c_{e}$, taking $\tau$ long enough makes electric propulsion, as regards specific impulse, better than rockets. Similarly, for long times, tethers are better than electric propulsion, whatever the relative values of ratios $c_{e} / 2 \eta_{e}$ and $v / \eta_{\text {eff }}$ in (1) and (2). In particular, a tether system with $\eta_{\text {eff }} \sim 1 / 3$ would be twice as light as the corresponding Ion-thruster for mission duration $\tau$ (weeks) $\sim \alpha(\mathrm{kg} / \mathrm{kW})$, which is about half a year, say, for a space tug, $\alpha \sim 25 \mathrm{~kg} / \mathrm{kW}$, and one month for ISS re-boost, $\alpha \sim 5 \mathrm{~kg} / \mathrm{kW}[26]$.

\section{LWT model in thruster mode}

\subsection{General considerations}

The left scheme in Fig. 1 shows a tether configuration in thrust mode for a mission in an eastward, low inclination orbit [24]. The tether has anodic and cathodic segments for electron collection and emission, with lengths $L_{a}$ and $L_{c}$, which are deployed downwards and upwards, respectively. An insulated segment of length $L_{i}$ is located between a power supply at the satellite and the bare anodic segment in order to increase efficiency; in a fully bare tether, $L_{i}=0$, electrons collected near the top, where bias is largest, would do little push work but would fully consume energy from the power source. The satellite, which is schematically represented by a box with a battery inside, should provide an electromotive force $\mathcal{E}$ (volts). The middle and right panels, not drawn in scale, show a scheme of the current and voltage profiles. The bare tether, which is positively polarized with respect to the plasma, works as a giant Langmuir probe and captures electrons, mostly at high bias. Electrons are emitted back to the plasma by the negatively polarized segment through thermionic and photoelectric effects due to the low-W coating.

Thanks to the good and steady electrical contact with the ionospheric plasma provided by the configuration of Fig. 1, a current $\boldsymbol{I}=I(x) \boldsymbol{u}_{t}$ circulates along the tether and generate the Lorentz force

$\boldsymbol{F}_{M}=\int_{0}^{L} \boldsymbol{I} \times \boldsymbol{B} d x \approx\left(\boldsymbol{u}_{t} \times \boldsymbol{B}\right) \int_{0}^{L} I(x) d x \equiv L I_{a v}\left(\boldsymbol{u}_{t} \times \boldsymbol{B}\right)$,

where we ignored magnetic field variations along the tether and took it as a rigid dumbbell perfectly aligned with the local vertical of the orbit. We also used the total tether length

$\frac{L_{i}}{L}+\frac{L_{a}}{L}+\frac{L_{c}}{L} \equiv \tilde{L}_{i}+\tilde{L}_{a}+\tilde{L}_{c}=1$

and the unit vector $\boldsymbol{u}_{t}$ along the current direction that points from $C$ to $A$ in Fig. 1. If we introduce $E_{m} \equiv \boldsymbol{u}_{t} \cdot\left(\boldsymbol{v}_{\text {rel }} \times \boldsymbol{B}\right)$ and the orbital velocity $\boldsymbol{v}$, with $\boldsymbol{v}_{r e l} \approx \boldsymbol{v}$ for the slow ionospheric plasma corotating with the Earth, we conclude that thrust generation then requires

$W_{M} \equiv \boldsymbol{F}_{M} \cdot \boldsymbol{v}=-E_{m} L I_{a v}>0$

indicating that the current should flow in the opposite direction of the motional electric field $\boldsymbol{v}_{r e t} \times \boldsymbol{B}$, the mission requiring $E_{m}<0$; a non-relativistic $\left(v_{r e l}<<\right.$ light velocity $c$ ) Lorentz transformation readily shows that $\boldsymbol{v}_{\text {rel }} \times \boldsymbol{B}$ is the electric field in the highlyconductive ionospheric background plasma, for an observer moving with the tether. Ultimately, current flows in the opposite direction of the naturally induced electric field as a result of the power $W_{E}=\mathcal{E} I_{B}$ supplied at the satellite at the satellite, with $I_{B}$ the current at points $B^{+}$and $B^{-}$.

In the configuration shown in Fig. 1, we assumed that the electromotive force $\mathcal{E}$ is large enough to have $\Phi(x)>0$ and $\Phi(x)<0$ in the anodic and insulated $\left(A A^{*}\right.$ and $\left.A^{*} B^{+}\right)$, and coated $\left(B^{-} C\right)$ segments, respectively. Here $\Phi(x)=\Phi_{t}-\Phi_{p l}$ is the local bias difference between tether and faraway ambient plasma, increasing from $A$ to $B^{+}$, and being less negative from $B^{-}$to $C$. At both tether ends we impose the boundary conditions $I_{A}=I_{C}=0$ (due to $w_{t} h_{t} \ll<w_{t} L$, with $h_{t}$ the tether thickness or radius, current collection and emission at both tether tips is fully negligible). As regards bias at the ends, note an important difference: bias should keep negative from $B^{-}$to $C$, i-e., $\Phi_{C}$ should not be positive; an electron-attracting segment at end $C$ of the tether would increase current, not allowing to satisfy condition $I_{C}=0$. An ion-attracting segment at end $A$, on the other hand, would have a negative, though insignificant, effect on the circuit.

The tether can be considered as a system that converts electrical power $W_{E}$, from solar panels or batteries in Fig. 1, into mechanical power $W_{M}$. System efficiency $\eta_{\text {eff }}$, introduced in Eq. (2) to conceptually present the tether as part of the power plant, can be also conveniently rewritten as

$\frac{1}{\eta_{\text {eff }}}=\frac{W_{E}+\alpha_{t} m_{t} / \alpha}{W_{M}}$ 
explicitly involving, as opposite to Eq. (2), twice the desired mechanical power rather than the supply power, visualizing effective conversion efficiency. This will be later shown to help in system design considerations. System efficiency $\eta_{e f f}$ directly decreases with increased supply power $\varepsilon I_{B}$, which might be required to have $\Phi_{C}$ negative, and increases with increasing magnetic power per unit mass $\alpha W_{M} / \alpha_{t} m_{t}$. Calculations leading to system efficiency require evaluation of current profiles to determine both the Lorentzdrag power

$\frac{W_{M}}{\left|E_{m}\right|}=I_{a \nu} L=\int_{0}^{x_{A^{*}}} I(x) d x+\int_{x_{A^{*}}}^{x_{B}} I(x) d x+\int_{x_{B}}^{L} I(x) d x$

and the supply power $W_{E}=\mathcal{E} I_{B}$.

\subsection{Current-voltage profiles}

Detemination of system efficiency requires solving current and voltage profiles all along the tether, depending on design parameters and orbital conditions. In a reference frame tied to the tether, the ionospheric plasma is subject to the motional electric field. Faraway plasma potential $\Phi_{p t}$ is then given by its gradient $d \Phi_{p l} / d x=$ $E_{m}<0$. Current and potential inside the tether satisfy 0 hm's law $J(x)=\sigma_{t} A_{t} d \Phi_{t} / d x$. The equation for the local bias $\Phi=\Phi_{t}-\Phi_{p t}$ valid throughout, is

$\frac{d \Phi}{d x}=\frac{I(x)}{\sigma_{t} A_{t}}+\left|E_{m}\right|$

Regarding current profile, if tether width (or radius) is not too large, the anodic segment $(\Phi>0)$ will collect electrons following the orbital-motion-limited (OML), high bias law [31]

$j_{O M L}(\Phi)=\frac{e N_{0}}{\pi} \sqrt{\frac{2 e \Phi}{m_{e}}}$

with $e$ the elementary charge, $m_{e}$ the electron mass, and $N_{0}$ the ambient plasma density. On the other hand, if $\Phi$ is negative enough to avoid space charge effects (a requirement discussed in Sec. 3.4), the emission in the cathodic segment $(\Phi<0)$ is biasindependent and given by [22]

$$
\begin{aligned}
j_{c}= & j_{t h}+j_{p h}=A T_{t}^{2} \exp \left(-\frac{W_{t}}{k_{B} T_{t}}\right) \\
& +f\left(1-r_{t}\right) e \int_{0}^{\infty} S(E) Y_{p h}(E) d E .
\end{aligned}
$$

For the thermionic current density $j_{t h}$, we have the RichardsonDushman law [32] with $A \approx 1.2 \times 10^{5} \mathrm{~A} / \mathrm{m}^{2} \mathrm{~K}^{2}$, and $W_{t}$ and $T_{t}$ the tether work function and temperature, respectively. Regarding the photoelectric current density $j_{p h}$ it involves the energy spectrum of the solar photons ( $S$ ) with energy $E$, the tether reflectivity $\left(r_{t}\right)$ and photoelectric yield $\left(Y_{p h}\right)$, and a factor $f$ that takes into account that only a fraction of the tether perimeter is illuminated. We then have

$\frac{d I}{d x}= \begin{cases}p_{t} \text { jomi } & 0<x<x_{A^{*}} \\ 0 & x_{A^{*}}<x<x_{B} \\ -p_{t} j_{C} & x_{B}<x<x_{C}\end{cases}$

where $p_{t}$ is the tether perimeter and we wrote $B^{-}=B^{+}=B$. The integration of Eqs. (9) and (12) with the boundary conditions $I_{A}=$ $I_{C}=0$ gives the current and voltage profiles that are necessary to compute $W_{E}, W_{M}, I_{a v}$ and $\eta_{e f f}$.

\subsubsection{Cathodic and insulated segments}

Analysis of thermionic and insulated segments is immediate because they do not involve bias $\Phi$ profiles when no space charge effect occurs throughout the cathodic segment. Middle and lower equations in (12) are immediately integrated with the boundary condition $I_{C}=0$ to give

$$
\begin{array}{ll}
I(x)=I_{B} \equiv j_{C} p_{t} L_{C} & x_{A^{*}}<x<x_{B} \\
I(x)=I_{B}-j_{c} p_{t}\left(x-x_{B}\right) & x_{B}<x<x_{C}
\end{array}
$$

Therefore, if the electromotive force provided by the power supply is high enough (see Sec, 3.4), the maximum current $I_{B}=j_{c} p_{i} L_{t}$ just depends on the characteristics of the cathodic segment independently of the electromotive force itself. These equations can now be used to allow direct computation of bias profile from $A^{*}$ to $C$, required for determining the battery-voltage range ensuring that bias $\Phi_{C}$ is negative enough. Using Eqs. (13) and (14) in Eq. (9) gives

$$
\begin{gathered}
\Phi_{C}-\Phi_{B^{-}}=\left|E_{m}\right| L_{c}\left(1+\frac{j_{c} p_{t} L_{c}}{2 \sigma_{t}\left|E_{m}\right| A_{t}}\right) \\
\Phi_{B^{+}}-\Phi_{A^{*}}=\left|E_{m}\right| L_{i}\left(1+\frac{j_{c} p_{t} L_{c}}{\sigma_{t}\left|E_{m}\right| A_{t}}\right)
\end{gathered}
$$

Across the power source we would just have

$\Phi_{B^{+}}-\Phi_{B^{-}}=\hat{\mathcal{E}}$

\subsubsection{Anodic segment}

Results for current and bias profiles at the anodic segment are readily obtained following well established analysis of tether OML collection [20]. After introducing characteristic magnitudes $I_{*} \equiv \sigma_{t}\left|E_{m}\right| A_{t}$ (short circuit current), $L_{*}=\ell^{1 / 3} h_{t}^{2 / 3}$, and $\ell \equiv$ $9 \pi^{2} m_{e} \sigma_{t}^{2}\left|E_{m}\right| / 128 e^{3} N_{0}^{2}$, and variables $i \equiv I / I_{*}, \phi \equiv \Phi /\left|E_{m}\right| L_{*}$ and $\xi \equiv x / L_{*}$, Eq. (9) and upper (12) become

$$
\begin{aligned}
& \frac{d \phi}{d \xi}=i+1 \\
& \frac{d i}{d \xi}=\frac{3}{4} \sqrt{\phi}
\end{aligned}
$$

with $h_{t} \approx 2 A_{t} / p_{t}$ the tether thickness for a tape (or the radius for a round tether). The above system admits the first integral $2 i+$ $i^{2}-\phi^{3 / 2}=$ const and should satisfy the boundary conditions $i(0) \equiv$ $i_{A}=0$ and

$i\left(x_{B}\right)=i_{B}=\frac{j_{c} p_{t} L_{c}}{\sigma_{t}\left|E_{m}\right| A_{t}} \equiv \tilde{j}_{c} \tilde{L}_{c}$

with $\tilde{j}_{c} \equiv j_{c} p_{t} L / \sigma_{t}\left|E_{m}\right| A_{t}$ and $\tilde{L}_{c}=L_{c} / L$. Tether thickness $h_{\mathrm{t}}$ appearing in both $j_{c} \sim p_{t} / A_{t} \approx 2 / h_{t}$ and $L_{*}=\ell^{1 / 3} h_{t}^{2 / 3}$, affect both the cathodic and anodic contacts. With the boundary conditions, the above first integral gives

$\phi_{A^{*}}\left(\phi_{A}, i_{B}\right)=\left(\phi_{A}^{3 / 2}+2 i_{B}+i_{B}^{2}\right)^{2 / 3}$.

and used in Eq. (18) leads to

$\frac{\tilde{L}_{a}}{\tilde{L}_{*}}=\int_{\phi_{A}}^{\phi_{A^{*}}} \frac{d \phi}{\sqrt{1+\phi^{3 / 2}-\phi_{A}^{3 / 2}}}$

Equations (21) and (22) determine dimensionless values $\phi_{A}$ and $\phi_{A^{*}}$ in terms of ambient and tether-design parameters. Fig. 2 shows $\tilde{L}_{a} / \tilde{L}_{*}$ versus $\phi_{A}$ for several values of $i_{B}$. It explicitly shows that the value of the maximum current in the tether $i_{B}$ can exceed 


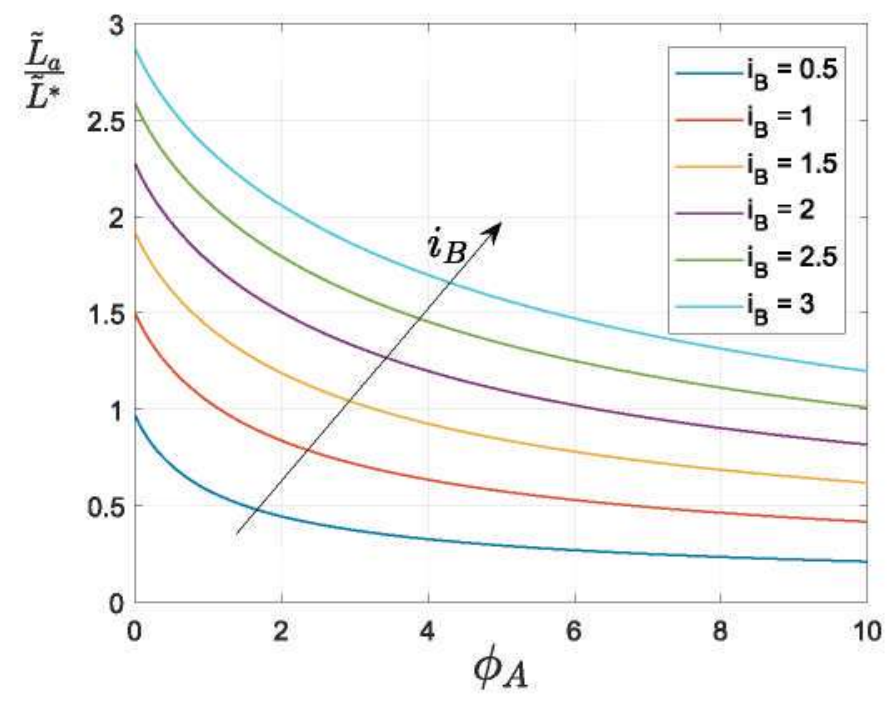

Fig. 2. $\tilde{L}_{a} / \tilde{L}_{*}$ versus $\phi_{A}$ for several $i_{B}$ values.

unity for sufficiently high power supply and design of the cathodic segment. This is a key difference with respect to a tether in passive mode (deorbiting scenarios without power supply), where the maximum current should be below the short circuit current. An alternative form to Eq. (22) that is more appropriate for fast numerical calculations, for instance during orbit propagation, is given in Appendix A.

\subsection{Overall LWT efficiency}

From Eq. (8), and current profiles obtained in Sec. 3.2, one finds the normalized mechanical power

$$
\begin{aligned}
\tilde{W}_{M} & \equiv \frac{W_{M}}{\sigma_{t} E_{m}^{2} A_{t} L}=\frac{I_{a v}}{I_{*}} \equiv i_{a v} \\
& =L_{*}\left(\phi_{A^{*}}-\phi_{A}\right)-\left(1+i_{B}\right) \tilde{L}_{a}+i_{B}\left(1-\frac{\tilde{L}_{c}}{2}\right)
\end{aligned}
$$

in terms of 4 dimensionless ratios, $\tilde{j}_{c}, \tilde{L}_{*}, \tilde{L}_{c}$ and $\tilde{L}_{a}$. Remember that, from Eq. (20), $i_{B}=\tilde{j}_{c} \tilde{L}_{c}$. We note that the first 2 terms in (23) can be readily rewritten, using (21) and (22), as the product of $\tilde{L}_{*}$ times a function of just $\phi_{A}$ and $\phi_{A^{*}}$, that is always negative, whereas $W_{M}$ must be positive. This directly points to the convenience of system design involving a small $L_{*} / L$ ratio, as in thrusting with HC tether-plasma cathodic contact [26].

Similarly, adding Eqs, (15)-(17) and using $W_{E}=I_{B} \mathcal{E}$ yield the normalized electrical power

$$
\begin{aligned}
\tilde{W}_{E} & =\frac{I_{B}}{\sigma_{t}\left|E_{m}\right| A_{t}} \frac{\mathcal{E}}{\left|E_{m}\right| L}=i_{B} \tilde{\mathcal{E}} \\
& =i_{B}\left[\left(\phi_{A^{*}}-\phi_{C}\right) \tilde{L}_{*}-\left(1+i_{B}\right) \tilde{L}_{a}+i_{B}\left(1-\frac{1}{2} \tilde{L}_{c}\right)+1\right]
\end{aligned}
$$

in terms of the same 4 dimensionless ratios above, except for the $\phi_{C}$ term. Of course, $\mathcal{E}$ is a design parameter free to select as long as $\phi_{C}$ comes out negative, efficiency being larger the smaller is $\mathcal{E}$. Setting $\phi_{C}=0$ in (24) would give the minimum $\mathcal{E}$ and minimum power $W_{E}=\mathcal{E} \times \sigma_{t}\left|E_{m}\right| A_{t} \times i_{B}$ allowed for any particular parameter-arrangement considered, making possible system design in looking for maximum performance. Use of $m_{t}=\rho_{t} A_{t} L$ and Eqs. (23) and (24) immediately determines the overall efficiency of a tether thruster defined in Eq. (2), rewritten in Eq. (7) as $\frac{1}{\eta_{e f f}}=\frac{W_{E}}{W_{M}}+\frac{\alpha_{t} \rho_{t} A_{t} L}{\alpha W_{M}}=\frac{\tilde{W}_{E}}{\tilde{W}_{M}}+\frac{1 / \tilde{E}_{m}^{2}}{\tilde{W}_{M}}$

with $\tilde{E}_{m} \equiv E_{m} \sqrt{\alpha \sigma_{t} / \alpha_{t} \rho_{t}}$.

\subsection{Minimum electromotive force for avoiding space-charge effects}

The dimensionless electromotive force $\tilde{\mathcal{E}}$ is a design parameter free to select. However, there are certain constraints that should be considered in order to make the assumptions of our model valid. Equation (24) shows us that $\tilde{\mathcal{E}}=\tilde{\mathcal{E}}_{1}^{m i n}-\tilde{L}_{*} \phi_{\mathrm{C}}$ with

$\tilde{\mathcal{E}}_{1}^{\text {min }} \equiv \tilde{L}_{*} \phi_{A^{*}}-\left(1+i_{B}\right) \tilde{L}_{a}+i_{B}\left(1-\frac{\tilde{L}_{C}}{2}\right)+1$.

Therefore, a first condition is $\tilde{\mathcal{E}}>\tilde{\mathcal{E}}_{1}^{\text {min }}$ in order to have $\phi_{C}<0$, as required to satisfy condition $I_{C}=0$. Moreover, since $j_{c}$ does not depend on the bias, a larger value of $\tilde{\mathcal{E}}$ will just support a corresponding uniformly-larger negative bias throughout the segment $\mathrm{BC}$, with no change in bias through the anodic and insulated segments, and changes in current nowhere in the tether. Therefore, setting $\tilde{\mathcal{E}}=\tilde{\mathcal{E}}_{1}^{\text {min }}$ would give the minimum electrical power allowed for any particular parameter arrangement considered, making possible system design in looking for maximum performance.

However, the choice $\tilde{\mathcal{E}}=\tilde{\mathcal{E}}_{1}^{\min }$ would not be consistent with lower Eq. (12) in our model. When we write $d I / d x=-p_{t} j_{c}$, with $j_{c}$ bias-independent, we are assuming that the bias is negative enough for the potential to increase monotonically away from the emitting material in the plane normal to the tether, otherwise space charge from the emitted electrons results in a local potential minimum that reduces emission itself, pushing emitted electrons not energetic enough back to the tether. If we ignore for simplicity the photoelectrons, the approximate (find a discussion in Ref. [33]) condition to reach a monotonic potential is

$\frac{e\left|\Phi_{C}\right|}{k_{B} T_{e}}>0.39\left(\frac{N_{e m}}{N_{0}}\right)^{1.4}$

with $T_{e}$ the electron temperature of the unperturbed plasma and $N_{e m}$ the density of the emitted electrons. The latter is directly related with $j_{t h}$ if we assume a half-Maxwellian emitted distribution function, thus yielding $j_{t h}=e N_{e m} \sqrt{2 k_{B} T_{t} / \pi m_{e}}$. Using this result in the relation $\tilde{\mathcal{E}}=\tilde{\mathcal{E}}_{1}^{\text {min }}-\tilde{L}_{*} \phi_{C}$ gives us

$\tilde{\mathcal{E}}_{2}^{\text {min }}=\tilde{\mathcal{E}}_{1}^{\min }+0.39 \frac{k_{B} T_{e}}{e\left|E_{m}\right| L}\left(\frac{j_{t h}}{e N_{0}} \sqrt{\frac{\pi m_{e}}{2 k_{B} T_{t}}}\right)^{1.4}$.

If $\tilde{\mathcal{E}}>\tilde{\mathcal{E}}_{2}^{\min }$, then the LWT operates free of space charge effects and without developing an anodic segment at $B^{-} C$. A design value $\tilde{\mathcal{E}}<\tilde{\mathcal{E}}_{2}^{\min }$ would require splitting analysis of the cathodic segment into 2 sub-segments, with RD emission in one, space charge limited emission applying in the other [34]. Actually, in characteristic tether applications, say $\left|E_{m}\right| \sim 150 \mathrm{~V} / \mathrm{km}, T_{e} \sim 0.1 \mathrm{eV}$, and $L \sim 5 \mathrm{~km}$, one finds $0.39 k_{B} T_{e} / e\left|E_{m}\right| L \sim 5.2 \times 10^{-5}$. Therefore, $\phi_{C}$ will be comparatively very small for typical values of both $N_{e m} / N_{0}$ and $L / L_{*}$. For that reason, in the analysis of Sec. 4.1 we will reasonably take $\tilde{\mathcal{E}}=\tilde{\mathcal{E}}_{1}^{\text {min }}$, whereas the full Eq. (28) might be required at numerical analysis, as in Sec. 4.2 , for extreme particular conditions. Note that our writing $h_{t} \approx 2 A_{t} / p_{t}$ involves neglecting thickness-to-width ratios of order $10^{-3}$ in typical applications of bare tape-tethers.

\section{High-efficiency design of LWT in thrust mode}

This section discusses the optimal design of a LWT in thrust mode. The proposed scheme is based on the maximization of the 
efficiency introduced in Eq. (7) together with rational mission constraints like for instance the required thrust level. Two scenarios are considered: the application of a LWT to re-boost a large space station with a power plant available and independent of the thrust and a tug-thruster mode. For convenience, we first get insight in the optimization problem by performing an analytical analysis in the long-tether limit approximation. The general case is studied numerically in Sec. 4.2.

\subsection{The long-tether limit}

Setting $\phi_{C}=0$, i.e. $\tilde{\mathcal{E}}=\hat{\mathcal{E}}_{1}^{\text {min }}$, Eq. (24) takes the extremely simple form $\tilde{W}_{E}=i_{B}\left(\tilde{W}_{M}+\phi_{A} \tilde{L}_{*}+1\right)$. Efficiency in Eq. (25) then becomes

$\frac{1}{\eta_{e f f}}=i_{B}+\frac{i_{B}\left(1+\phi_{A} \tilde{L}_{*}\right)+\tilde{E}_{m}^{-2}}{\tilde{W}_{M}}$

with the mechanical power rewritten as $\tilde{W}_{M}=\left(1+\tilde{L}_{i}\right) i_{B} / 2+$ $\tilde{L}_{*} G\left(\phi_{A}, i_{B}\right)$ by using (5) to replace $\tilde{L}_{C}$ in Eq. (23) and function $G$ defined and discussed in Appendix B.

We will now look for optimal perfomance conditions, considering small values of the $L_{*} / L$-ratio (long tether limit), as suggested below Eq. (23), and ignore $\tilde{L}_{*}$ terms in the above form of $\tilde{W}_{M}$ and in Eq. (29), which now reads

$\frac{1}{\eta_{e f f}} \approx i_{B}+\frac{2\left(i_{B}+\tilde{E}_{m}^{-2}\right)}{i_{B}\left(1+\tilde{L}_{i}\right)}$

as function of both ambient $\tilde{E}_{m}$-parameter and design $\tilde{L}_{\mathrm{i}}$-parameter and related value $i_{B}$. While efficiency in Eq. (30) just increases with $\tilde{L}_{i}$ within the valid range $0<\tilde{L}_{i}<1$, condition $\partial \eta_{\text {eff }} / \partial i_{B}=0$ shows that it does reach a maximum as function of $i_{B}$,

$\eta_{e f f}^{*}=\frac{1+\tilde{L}_{i}}{2\left(1+\sqrt{2 \tilde{E}_{m}^{-2}\left(1+\tilde{L}_{i}\right)}\right)}$

at the value $i_{B}^{*} \equiv \sqrt{2 \tilde{E}_{m i}^{-2} /\left(1+\tilde{L}_{i}\right)}$. We first note that the efficiency in Eq. (31) tops a maximum, $\tilde{E}_{m} /\left(\tilde{E}_{m}+2\right.$ ) at $\tilde{L}_{j}=1$, as in the case of using a $\mathrm{HC}$ for cathodic contact [26]. Actually, having $\tilde{L}_{j}$ near unity, i.e. having negligibly short anodic and cathodic segments ( $A A^{*}$ and $B C$ in Fig. 1, respectively) will most often be far from convenient or possible. Although having a high efficiency, the mechanical power obtained from the system would be small. Consider then how efficiency decreases as $\tilde{L}_{i}$ is cut in half, from 1 to 0.5 , a half-range covering insulated-segment lengths that lead to highest efficiencies. At $\tilde{L}_{i}=0.5$, Eq. (31) gives $\eta_{e f f}^{*}=0.75 \tilde{E}_{m} /\left(\tilde{E}_{m}+\sqrt{3}\right)$. The resulting efficiency drop is less than $20 \%$ for representative $\tilde{E}_{m}$ values, from 0.429 to 0.348 for $\bar{E}_{m}=1.5$, and from 0.273 to 0.227 for $\tilde{E}_{m}=0.75$. Actually, the full efficiency drop to $L_{j}=0$ is less than $40 \%$

We further note that $i_{B}^{*}$ can also be used to write $\tilde{L}_{j}$ as

$\tilde{L}_{f}=\frac{2}{\left(\tilde{E}_{m} i_{B}^{*}\right)^{2}}-1$

for convenient determination of the $i_{B}^{*}$ range of values for different ambient and design parameters. The range $0.5<\tilde{L}_{i}<1$ above implies condition

$2 / \sqrt{3}>\tilde{E}_{m} i_{B}^{*}>1$
For $E_{m}=150 \mathrm{~V} / \mathrm{km}$ (a representative value in $\mathrm{LEO}$ orbits at low inclination) and $\alpha=20 \mathrm{~kg} / \mathrm{kW}$ (at the tug-thruster mode), we would have $\tilde{E}_{m}=1.5$ for aluminum tethers, with factor $\alpha_{t}=2.5$ for tether-related hardware. Equation (33) then reads

$0.77 \approx 4 / 3 \sqrt{3}>i_{B}^{*}>2 / 3 \approx 0.67$

Moving to $\alpha=5 \mathrm{~kg} / \mathrm{kW}$, when a power plant is available independently of thrusting (case of the ISS), $\tilde{E}_{m i}$ decreases to 0.75 , the $i_{B}^{*}$ bounds in Eq. (34) increasing by a factor of 2 . A similar result applies for $\alpha$ back to the tug-mode but considering LEO orbits at moderately high inclination, with $E_{m}$ down to $75 \mathrm{~V} / \mathrm{km}$, say. For combined moderately-high inclination and $\alpha=5 \mathrm{~kg} / \mathrm{kW}, i_{B}^{*}$ bounds in Eq. (34) would increase by a factor 4.

Typical values for $i_{B}^{*}$ will be used in Appendix B to discuss validity of the approximation of $\mathrm{Eq}$. (30). Here we discuss how such values could be used as help in selecting the fractional lengths of all three tether segments, in the long-tether approximation, compatible with Eq. (5). In order to gain insight into the problem, the study is carried out for the particular case $\phi_{A}=1$ because the ratio $\tilde{L}_{\mathbf{a}} / \tilde{L}_{*}$ takes a very simple analytical form (see Eq. (43) and consider Fig. 2 to extend the results to other $\phi_{A}$ values). For $\phi_{A}=1$, Eq. (5) can be written as

$\left(\frac{2}{\left(\tilde{E}_{m} i_{B}^{*}\right)^{2}}-1\right)+4\left[\left(1+i_{B}^{*}\right)^{1 / 3}-1\right] \tilde{L}_{*}+\frac{i_{B}^{*}}{\tilde{j}_{c}}=1$

where $i_{B}^{*}$ is itself related to $\check{E}_{m}$ as discussed above. In Eq. (35) we used Eq. (20) and simple results from Eqs. (21) and (22) for $\phi_{A}=1$,

$\frac{\tilde{L}_{a}}{\tilde{L}_{*}}=4\left(\phi_{A}^{* 1 / 4}-1\right)=4\left[\left(1+i_{B}\right)^{1 / 3}-1\right]$

Note the simple relation between anodic and cathodic lengths, independent of both Eq. (30) and current $i_{B}$ though varying with the actual $\phi_{A}$ value,

$\left(1+\frac{\tilde{L}_{a}}{4 \tilde{L}_{*}}\right)^{3}=1+\tilde{j}_{c} \tilde{L}_{c}$

Consider low orbital inclination $\left(E_{m}=150 \mathrm{~V} / \mathrm{km}\right.$ and no dedicated power plant $(\alpha=5 \mathrm{~kg} / \mathrm{kW})$, leading to $\bar{E}_{m}=0.75$ above and $i_{B}^{*}$ bounds in Eq. (34) increasing by a factor of $2, i_{B}^{*}=1.54$ for $\tilde{L}_{i}=0.5$. To determine $\tilde{L}_{c}$ we use values $W_{t}=1.5 \mathrm{eV}$ and $T_{t}=580 \mathrm{~K}=0.05 \mathrm{eV}$ in Richardson-Dushman law, with ratio $W_{t} / k_{B} T_{t}=30$; also, we use aluminum conductivity, and values $L=10 \mathrm{~km}$ and $h_{t}=30 \mu \mathrm{m}$, finally yielding $\tilde{j}_{c} \approx 4.7, \hat{L}_{C}=i_{B}^{*} / \bar{j}_{R D} \approx$ 0.33 . Using $i_{B}^{*}=1.54$ in $\tilde{L}_{a}$, at $\mathrm{Eq}$. (36), we first get $\tilde{L}_{a} / \tilde{L}_{*} \approx 1.46$, then $\tilde{L}_{a}=1-0.5-0.33 \approx 0.17$, and $\tilde{L}_{*} \approx 0.17 / 1.46 \approx 0.116$. Moving to $E_{i n}=120 \mathrm{~V} / \mathrm{km}$ (a proper value to analyze ISS-re-boost at mid-inclinations) could keep the above results if $L$ is reduced to 8 $\mathrm{km}$, which is actually more convenient.

\subsection{General regime}

Previous section provides useful physical insight on the optimal design of LWTs as thrusters. The assumptions used there for simplicity, such as small $\tilde{L}_{*}$, no photoelectric effects, and $\phi_{A}=1$, are now removed and performance computed numerically. In a general case, and besides tether photoelectric model, one readily finds that tether performance depends on the following mission-related parameters

$\tilde{E}_{m} \equiv E_{m} \sqrt{\frac{\alpha \sigma_{t}}{\alpha_{t} \rho_{t}}}, \quad \kappa \equiv \frac{2 j_{c}}{\sigma_{t}\left|E_{m}\right|}, \quad \gamma \equiv \frac{k_{B} T_{e}}{\mathrm{e}\left|E_{m}\right| \ell}$. 
$\nu \equiv \frac{N_{e m}}{N_{0}}=\frac{4 \kappa}{3} \sqrt{\frac{e\left|E_{m}\right| \ell}{\pi k_{B} T_{t}}}$

which basically involve ambient conditions and the state-of-the-art of LWT manufacturing. The design parameters are

$\tilde{h}_{t} \equiv \frac{h_{t}}{L}, \quad \tilde{L}_{*} \equiv \frac{L_{*}}{L}=\left(\frac{\ell}{L}\right)^{1 / 3} \tilde{h}_{t}^{2 / 3}, \quad \tilde{L}_{c} \equiv \frac{L_{C}}{L}$,

$\tilde{L}_{a} \equiv \frac{L_{a}}{L}, \quad \tilde{\mathcal{E}} \equiv \frac{\mathcal{E}}{\left|E_{m}\right| L}$

The optimal value of $\tilde{\mathcal{E}}$ avoiding space charge effects is $\tilde{\mathcal{E}}=\tilde{\mathcal{E}}_{2}^{\min }$, which will be taken hereafter. When evaluating $\tilde{W}_{E}=i_{B} \tilde{\mathcal{E}}$ and $\tilde{W}_{M}$ from Eq. (23), and the efficiency from (25), one should keep in mind that: (i) $\tilde{L}_{a}+\tilde{L}_{i}+\tilde{L}_{c}=1$, (ii) $\tilde{j}_{c}=\kappa / \tilde{h}_{t}$, (iii) $i_{B}=\tilde{j}_{c} \tilde{L}_{c}$, (iv) $\phi_{A^{*}}$ is given by Eq. (21), and (iv) $\phi_{A}, i_{B}$, and $\tilde{L}_{a} / \tilde{L}_{*}$ are linked by Eq. (22). Moreover, in order to meet the condition $\phi_{A}>0$, the length of the anodic segment should be below the threshold (see Appendix A)

$\tilde{L}_{a}^{\max } \equiv \frac{4}{3} \tilde{L}_{*} F_{S}\left[\cosh ^{-1}\left(1+i_{B}\right)\right]$

Otherwise, a cathodic segment ignored in our model would be developed. Therefore the design parameter $\tilde{L}_{a}$ should be within the range $0<\tilde{L}_{a}<\operatorname{minimum}\left(1-\tilde{L}_{c}, \tilde{L}_{a}^{\max }\right)$, at nominal environment; bias at $\mathrm{A}$ will often develop negative values through re-boost, with weak effects.

Hereafter we will consider typical values for a ISS re-boost mission, $E_{m}=120 \mathrm{~V} / \mathrm{km}$, at $51.64^{\circ}$ mid-inclination, $N_{0}=10^{11} \mathrm{~m}^{-3}$, $T_{e}=0.1 \mathrm{eV}$, and $\alpha=5 \mathrm{~kg} / \mathrm{kW}$, and the following tether parameters $\rho_{t}=2700 \mathrm{~kg} / \mathrm{m}^{3}, \sigma_{t}=3.546 \times 10^{7} \Omega^{-1} \mathrm{~m}^{-1}, \alpha_{t}=2.5$ and $W_{t}=1.5 \mathrm{eV}$. We will also adopt the tether photoelectric model presented in Ref. [22], which considers a Fowler-DuBridge law for the photoelectric yield. Since uncertainties exist now on the cathodic capabilities of LWTs, we will consider a hot case with $T_{t}=600 \mathrm{~K}$ and a cold case with $T_{t}=550 \mathrm{~K}$, which are compatible with the aluminum alloy $1100-\mathrm{H} 19$ (melting point at $923 \mathrm{~K}$ ). If a higher temperature would be needed due to difficulties on manufacturing a LWT with $W_{t}=1.5 \mathrm{eV}$, a BeCu alloy with melting point at $1300 \mathrm{~K}$ could be used. Regarding tether cross-section, we will consider a tape because it is well-known that it presents better performance than round tethers (find studies on current collection $[35,36]$, cut probability $[10,11]$ and dynamics [37] elsewhere). Table 1 summarizes the values of the dimensionless parameters.

Table 1

Parameters used in Sec. 4.2.

\begin{tabular}{lll}
\hline Parameter & Cold case & Hot case \\
\hline$\tilde{E}_{m}$ & 0.615 & 0.615 \\
$\kappa \times 10^{8}$ & 0.46 & 5.26 \\
$\gamma \times 10^{19}$ & 3.6 & 3.6 \\
$\nu$ & 8.3 & 91.9 \\
\hline
\end{tabular}

We start by analyzing the optimum values of the anodic $\left(\tilde{L}_{a}^{\text {opt }}\right)$, cathodic $\left(\tilde{L}_{c}^{o p t}\right)$, and insulated $\tilde{L}_{i}^{o p t}=1-\tilde{L}_{a}^{o p t}-\tilde{L}_{c}^{o p t}$ segments for a given tether thickness and length. The optimum is found by imposing the conditions $\partial \eta_{e f f} / \partial \tilde{L}_{a}=\partial \eta_{e f f} / \partial \tilde{L}_{c}=0$, i.e. looking for the maximum in a plot of $\eta_{e f f}$ in the $\tilde{L}_{a}-\tilde{L}_{c}$ plane. An example is given in Fig. 3, which corresponds to $h_{t}=30 \mu \mathrm{m}$ and $L=L_{*}$. The red lines correspond to $\tilde{L}_{a}+\tilde{L}_{c}=1\left(\tilde{L}_{i}=0\right)$ and $\tilde{L}_{a}=\tilde{L}_{a}^{\max }$ in Eq. (40) $\left(\phi_{A}=0\right)$, respectively. In this particular case (see red point in Fig. 3), one finds $\eta_{\text {eff }}^{\max } \approx 0.026$ for $\tilde{L}_{a}^{o p t} \approx 0.16$ and $\tilde{L}_{c}^{o p t} \approx 0.77$ (and insulated segment $\tilde{L}_{i} \approx 0.07$ ). Moving to the hot case (not shown) yields $\eta_{\text {eff }}^{\max } \approx 0.077$ for $\tilde{L}_{a}^{\text {opt }} \approx 0.54$ and $\tilde{L}_{c}^{\text {opt }} \approx 0.24$ (and insulated segment $\tilde{L}_{i} \approx 0.22$ ). Obviously, $\tilde{L}_{c}^{\text {opt }}$ decreases as the parameter $\kappa$

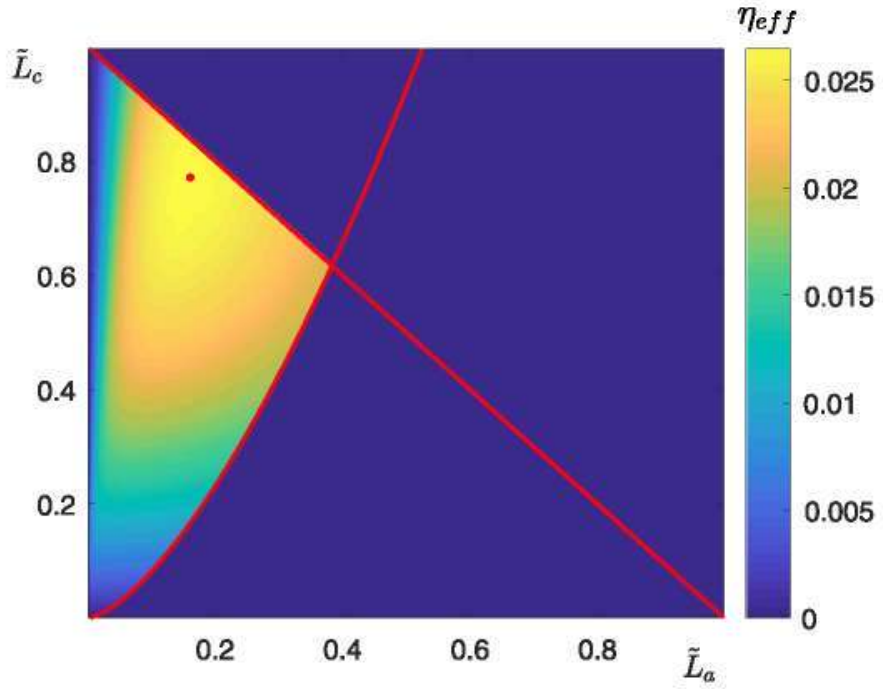

Fig. 3. Example of LWT efficiency in the $\tilde{L}_{a}-\tilde{L}_{c}$ plane. (For interpretation of the colors in the figure(s), the reader is referred to the web version of this article.)
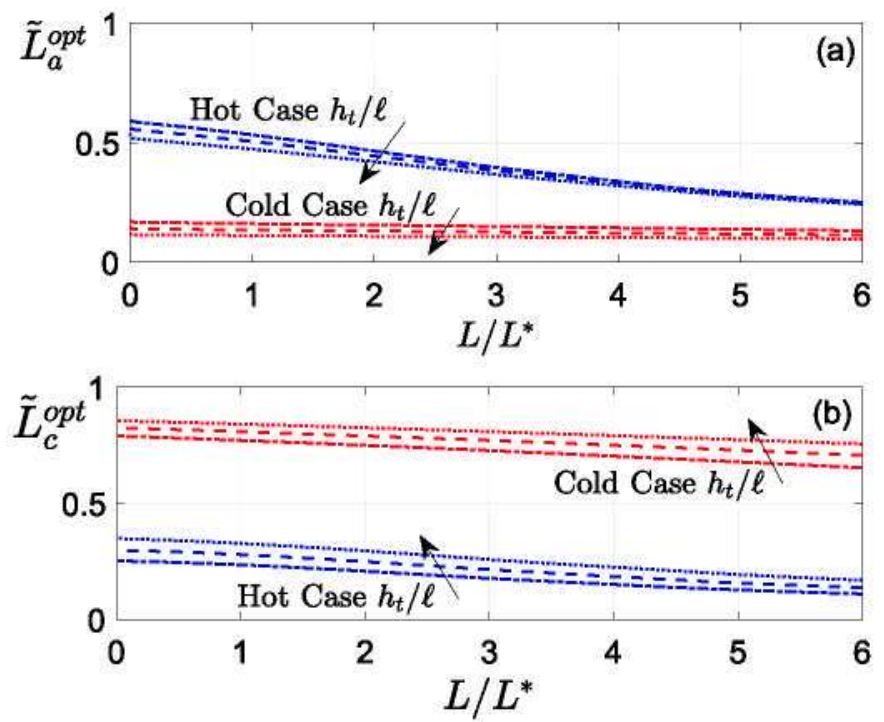

Fig. 4. Optimal tether lengths $\tilde{L}_{\tilde{G}}^{\text {opr }}$ (top) and $\tilde{L}_{c}^{\text {opr }}$ (bottom) versus $L / L_{*}$ for the parameters of Table 1 .

increases, i.e. the cathodic contact of the tether with the plasma is improved by lowering the work function-to-temperature ratio.

Following the previous procedure, i.e. imposing the conditions $\partial \eta_{e f f} / \partial \tilde{L}_{a}=\partial \eta_{e f f} / \partial \tilde{L}_{c}=0$, we computed $\tilde{L}_{a}^{o p t}\left(h_{t} / \ell, L / L_{*}\right)$ and $\tilde{L}_{c}^{\text {opt }}\left(h_{t} / \ell, L / L_{*}\right)$ for a range $0<L / L_{*}<6$ and $h_{t} / \ell \times 10^{23}$ equal to $1.3,2.6$ and 5.2 , which correspond to $h_{t} \approx 30,60$, and $120 \mu \mathrm{m}$ (note that $\ell=2.32 \times 10^{18} \mathrm{~m}$ for the values of Table 1). As shown in Fig. 4 , the relative lengths of the optimal anodic and cathodic segments decreases and increases with $h_{t} / \ell$ for a given $L / L_{*}$ ratio. The length of the optimal cathodic segment decreases with the temperature, and both the anodic and cathodic lengths decrease as $L / L_{*}$ increases. The latter is consistent with the analytical calculations of Sec. 4.1, where it was shown that the maximum efficiency in the long tether limit is reached for $\tilde{L}_{i} \rightarrow 1$.

If we now use the parameters of Table 1 and set $\tilde{L}_{a}=$ $\tilde{L}_{a}^{o p t}\left(h_{t} / \ell, L / L_{*}\right)$ and $\tilde{L}_{c}=\tilde{L}_{c}^{\text {opt }}\left(h_{t} / \ell, L / L_{*}\right)$, the optimal efficiency of a LWT in thruster mode can be found. Fig. 5 shows the efficiency versus $L / L_{*}$ for exactly the same $h_{t} / \ell$ ratios of Fig. 4 . For convenience, the optimal efficiency given by Eq. (31) with $\tilde{L}_{i}=1$, which coincides with the maximum efficiency of a bare tether with 


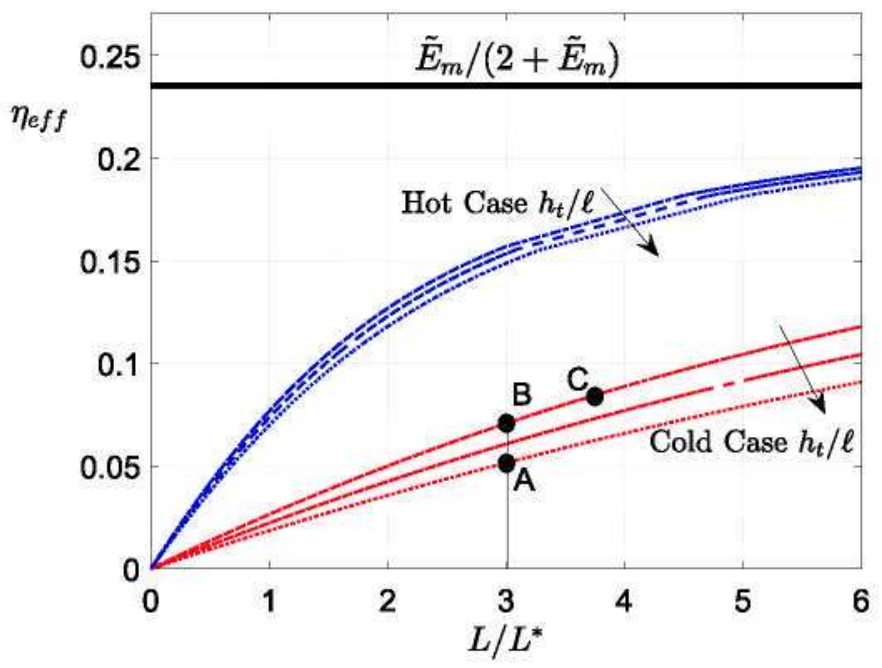

Fig. 5. LWT efficiency versus $L / L_{*}$ for the parameters of Table 1 .
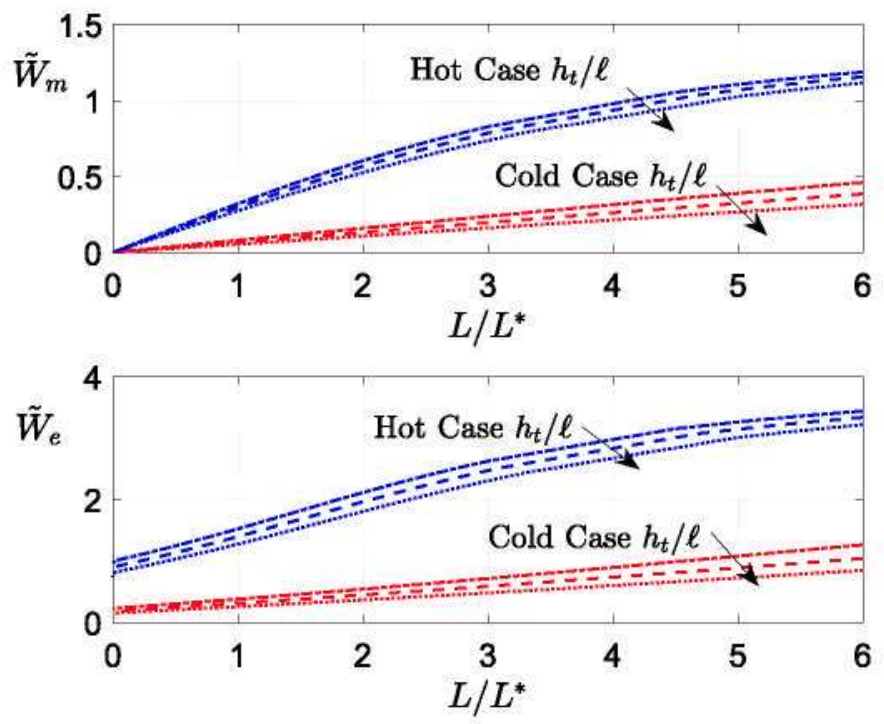

Fig. 6. $\tilde{W}_{m}$ and $\tilde{W}_{e}$ efficiency versus $L_{/} L_{*}$ for the parameters of Table 1.

an ideal HC, is also shown (black line). Clearly, LWT performance are very sensitive to the ratio $W_{t} / T_{t}$ : a temperature variation of $50 \mathrm{~K}$ changes the efficiency about a factor 3 . We also highlight that designing LWT missions with low thicknesses is doubly beneficial. First, since $\tilde{j}_{c} \sim h_{t}^{-1}$, decreasing the thickness and keeping $L / L_{*}$ fixed improves the efficiency because one moves from point $A$ to $B$ in Fig. 5 by improving the performance of the cathodic segment. This effect is less important in the hot case than in the cold case and vanishes as $\kappa \rightarrow \infty$. The behavior can be understood by remembering that the efficiency of a bare tether with $\mathrm{HC}$ does not involve $\tilde{h}_{t}$ when presented versus $L / L_{*}$ [26]. Second, for given length, decreasing the thickness also improves the performance of the anodic segment because $L / L_{*}=L / \ell^{1 / 3} h_{t}^{2 / 3}$. This is illustrated in Fig. 5 as a change from point $A$ to $C$. For completeness, Fig. 6 displays the normalized mechanical and electrical power for optimal lengths of the anodic and cathodic segments.

\section{Discussion and ISS application}

Previous results can be directly applied to re-boosting of the ISS. The optimal design of a LWT would require a deeper analysis involving not only the efficiency, as made in this work, but also other considerations as the air drag on the tether itself, tether cut probability considerations, and mechanical constraints, among others. However, we can give here some tentative values. The station, with mass about 420 tons, has an orbit with inclination $51.64^{\circ}$ and altitude around $400 \mathrm{~km}$. The aerodynamic drag $\boldsymbol{F}_{D}$, which is in the order of $0.2 \mathrm{~N}$, produces the decay of the station at a rate of $2 \mathrm{~km}$ per month (mechanical power $W_{M}^{D}=\boldsymbol{F}_{D} \cdot \boldsymbol{v} \approx 1.5 \mathrm{~kW}$ ). As already discussed, low tether thicknesses are beneficial but a lower bound may exist due to mechanical considerations. Let us take $h_{t}=30 \mu \mathrm{m}$ and the hot case in Table 1. Since the LWT should compensate the drag, tether width and length are linked by

$$
\begin{aligned}
w_{t}\left(L / L_{*}\right) & =\frac{W_{M}^{D}}{\sigma_{t} E_{m}^{2} h_{t} L_{*}} \times \frac{1}{\tilde{W}_{M}\left(L / L_{*}\right)} \frac{L_{*}}{L} \\
& =7.66 \mathrm{~cm} \times \frac{1}{\tilde{W}_{M}\left(L / L_{*}\right)} \frac{L_{*}}{L}
\end{aligned}
$$

where $\tilde{W}_{M}\left(L / L_{*}\right)$ is given in Fig. 6. Increasing the tether length, which produces higher efficiency according to Fig. 5, implies decreasing the width. However, the latter should be above some value to make the tether robust against small debris impacts [11]. Taking $w_{t}=2 \mathrm{~cm}$, that would make the anodic segment operate under the OML regime, one finds that Eq. (41) gives $L \approx 4 L_{*} \approx$ $5.11 \mathrm{~km}$ with $\tilde{W}_{M}=1.55 \mathrm{~kW}>W_{M}^{D}$. For that choice, the optimum lengths of the anodic, cathodic, and insulated segments are $L_{a}^{o p t} \approx 1.74 \mathrm{~km}, L_{c}^{o p t} \approx 0.77 \mathrm{~km}$, and $L_{i}^{o p t} \approx 2.6 \mathrm{~km}$. These numbers and Eq. (28) show that $\mathcal{E}_{1}^{\min } \approx \mathcal{E}_{2}^{\min }$ and, for the ISS case, one could safely take from the beginning $\mathcal{E}=\mathcal{E}_{1}^{\text {min }}$ and simplify the analysis. The efficiency of the system is $\eta_{\text {eff }} \approx 0.18$, the electrical power $W_{E} \approx 4.7 \mathrm{~kW}$, the mass of the tether $m_{t} \approx 8.3 \mathrm{~kg}$ and the mass of the tether system $m_{s} \approx 20.7 \mathrm{~kg}$.

The main advantage of the electrodynamic tether as compared with matured thrust technologies, including chemical and electrical thrusters, is its propellant-less character and, as shown in Sec. 2, its benefits increase with the duration of the mission. Regarding the comparison between LWTs and conventional bare tethers equipped with active electron emitters, a definitive conclusion cannot be found yet. The absence of an active electron emitter in the LWT system decreases its complexity considerably, reduces the power needs, and eliminates the needs for expellant. However, as shown in our analysis, LWT performance are very sensitive to the work function-to-temperature ratio and, the lower the ratio, the better the performance. A comparison among the two types of tethers requires knowing the results of on-going research activities on manufacturing and testing of LWT samples. Taking into account the melting point of alloys with high conductivity-to-density ratio, a value of the work function below around $2 \mathrm{eV}$ would be needed.

\section{Conclusions}

New advances in material science can open new opportunities for space tether technology. One of them is the LWT, a type of electrodynamic tether that does not need an active electron emitter. This work presented an electrical model for the analysis of LWT operation in thrust mode, where the electromotive force provided by an onboard power source is used to re-boost the spacecraft or compensate the air drag. The proposed scheme involves a bare anodic segment for electron collection, followed by an insulated segment, a power source, and a tether segment coated with a low work function material to enhance the passive electron emission through thermionic and photoelectric effects. The minimum electromotive force that is necessary to avoid the development of a tether segment operating under space charge conditions has been found, as well as key figures of merit of the system as a function of tether design parameters and ambient variables. 
Design conditions for high-efficiency operations have been studied analytically under sensible assumptions to simplify the equations. Tether efficiency, measuring the rate of conversion from electrical to mechanical power, increases with the ratio between the tether length and the characteristic length gauging ohmic effects. The optimal efficiency increases, though weakly, with the length of the insulated segment and a dimensionless parameter involving the motional electric field and the tether conductivityto-density ratio. Useful formulae relating the lengths of the anodic, cathodic and insulated segments with the maximum current value have been obtained.

For a general case, i.e. without taking any simplification in the proposed model, LWT performance was found numerically. For a given mission, and tether length and thickness, optimal lengths for the different tether segments were found and used to present optimal efficiencies. The results are very sensitive to the quality of the cathodic contact, which is mainly governed by the work functionto-temperature ratio. The analysis has been applied to the re-boost of the ISS. It indicates that a LWT of length, width and thickness equal to $5.1 \mathrm{~km}, 2 \mathrm{~cm}$, and $30 \mu \mathrm{m}$ can compensate the air drag of the station with no consumable if fed with a power source of $4.7 \mathrm{~kW}$.

\section{Declaration of competing interest}

This work was initially supported by Agencia Estatal de Investigación (Ministerio de Ciencia, Innovación y Universidades of Spain) under the project ESP2017-82092-ERC (AEI) and continued thanks to funding received from the European Unions Horizon 2020 research and innovation programme under grant agreement No. 828902 (E.T.PACK project). GSA is currently the Coordinator of E.T.PACK and co-author of a patent on low work function tethers in thrust mode:

G. Sanchez-Arriaga and C. Bombardelli. Orbit propulsion system by floating conductor cables, 05 2017. Spanish Patent WO2017081351A1.

The patent is cited in the bibliography of the manuscript.

\section{Acknowledgements}

This work was initially supported by Agencia Estatal de Investigación (Ministerio de Ciencia, Innovación y Universidades of Spain) under the project ESP2017-82092-ERC (AEI) and continued thanks to funding received from the European Unions Horizon 2020 research and innovation programme under grant agreement No. 828902 (E.T.PACK project). GSA work is supported by the Ministerio de Ciencia, Innovación y Universidades of Spain under the Grant RYC-2014-15357.

\section{Appendix A. The anodic segment}

Following a similar procedure to the one presented in Ref. [38] for bare tethers in passive modes, Eq. (22) can be written in a form that is more convenient for fast numerical calculations. For instance, for $\phi_{A}>1$, we write $1+i=\sqrt{\phi_{A}^{3 / 2}-1} \sinh v$ and find

$\frac{\tilde{L}_{a}}{\tilde{L}_{*}}=\frac{4}{3}\left(\phi_{A}^{3 / 2}-1\right)^{1 / 6} \int_{v_{s 0}}^{v_{s f}} \cosh ^{1 / 3} v d v$

A similar procedure for $\phi_{A}<1$ and $\phi_{A}=1$ yields

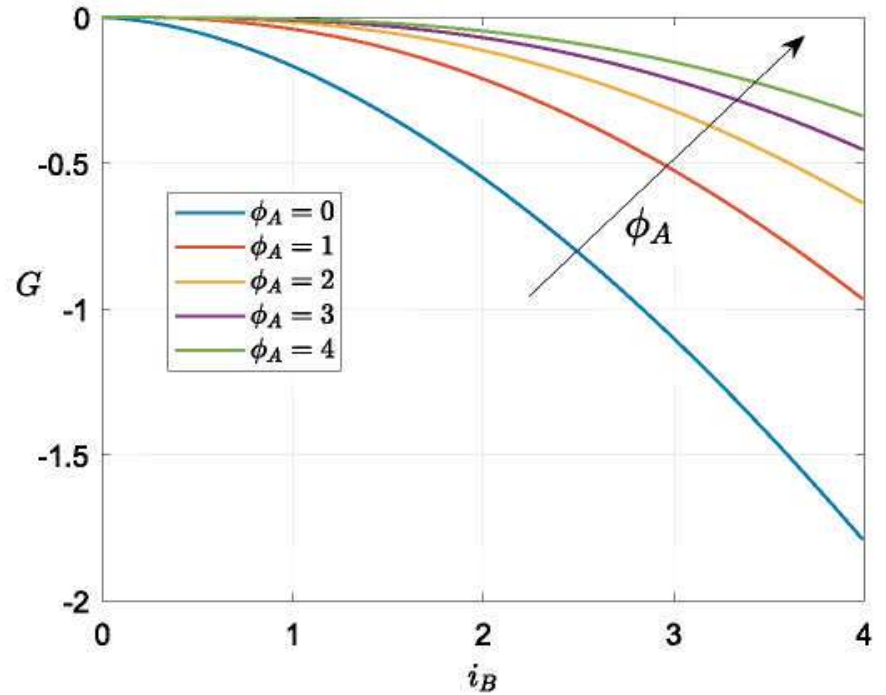

Fig. 7. Function $G$ versus $i_{B}$ for several $\phi_{A}$ values.

$$
\frac{\tilde{L}_{a}}{\tilde{L}_{*}}\left(\phi_{A}, i_{B}\right)= \begin{cases}\frac{4}{3}\left(1-\phi_{A}^{3 / 2}\right)^{1 / 6}\left[F_{S}\left(v_{c f}\right)-F_{S}\left(v_{c 0}\right)\right], & \phi_{A}<1 \\ 4\left[\left(1+i_{B}\right)^{1 / 3}-1\right], & \phi_{A}=1, \\ \frac{4}{3}\left(\phi_{A}^{3 / 2}-1\right)^{1 / 6}\left[F_{C}\left(v_{s f}\right)-F_{S}\left(v_{s 0}\right)\right], & \phi_{A}>1\end{cases}
$$

where

$F_{S}(x)=\int_{0}^{x} \sinh ^{1 / 3} \zeta d \zeta, \quad F_{c}(x)=\int_{0}^{x} \cosh ^{1 / 3} \zeta d \zeta$

and the variables $\cosh \left(v_{c f}\right)=\left(1+i_{B}\right) / \sqrt{1-\phi_{A}^{3 / 2}}, \cosh \left(v_{c 0}\right)=$ $1 / \sqrt{1-\phi_{A}^{3 / 2}}, \quad \sinh \left(v_{s f}\right)=\left(1+i_{B}\right) / \sqrt{\phi_{A}^{3 / 2}-1}, \quad \sinh \left(v_{s 0}\right)=1 /$ $\sqrt{\phi_{A}^{3 / 2}-1}$. This formulation is advantageous from a computational point of view because functions $F_{S}$ and $F_{C}$ are parameter-free and they can be also written explicitly in terms of hypergeometric functions.

\section{Appendix $B$. The function $G\left(\phi_{A}, i_{B}\right)$}

The dimensionless mechanical power could be written as $\tilde{W}_{M}=i_{B}\left(1+\tilde{L}_{i}\right) / 2+\tilde{L}_{*} G\left(\phi_{A}, i_{B}\right)$ with

$$
\begin{aligned}
G\left(\phi_{A}, i_{B}\right) & \equiv \int_{\phi_{A}}^{\phi_{A^{*}}}\left(1-\frac{1+\sqrt{1+\phi_{A^{*}}^{3 / 2}-\phi_{A}^{3 / 2}}}{2 \sqrt{1+\phi^{3 / 2}-\phi_{A}^{3 / 2}}}\right) d \phi \\
& \equiv \int_{\phi_{A}}^{\phi_{A^{*}}}\left[1-\frac{1+\left(i_{B} / 2\right)}{\sqrt{1+\phi^{3 / 2}-\phi_{A}^{3 / 2}}}\right] d \phi
\end{aligned}
$$

where we used again Eq. (5) and $\phi_{A^{*}}$ is given by Eq. (21). Dropping the term $\tilde{L}_{*} G\left(\phi_{A}, i_{B}\right)$ in the mechanical power, as done in Sec. 4.1, requires a discussion about function $G$. For particular test of consistency in our neglect of $L_{*} / L$ terms, consider the simple case of taking $\phi_{A}=1$ in Eqs. (21) and (22), G in Eq. (45) then reading

$G\left(i_{B}\right)=3+2 i_{B}-\left(3+i_{B}\right)\left(1+i_{B}\right)^{1 / 3}$

with $G$ increasing very slowly with $i_{B}: G\left(i_{B}<<1\right)=0\left(i_{B}^{3}\right), G(1) \approx$ $-0.04, G(2) \approx-0.21, G(3) \approx-0.52$, certainly yielding $\tilde{L}_{*}|G| \sim$ 
$\tilde{L}_{*} \ll<1$. This applies to the $i_{B}^{*}$ values considered in Sec. 4.1, ranging from 0.67 to $16 / 3 \sqrt{3} \approx 3.08$. Fig. 7 showing $G$ versus $i_{B}$ at several $\phi_{A}$ values, further confirms general consistency (beyond the particular $\phi_{A}=1$ case).

\section{References}

[1] J.C. Liou, A. Rossi, H. Krag, M. Xavier, James Raj, K. Anilkumar, T. Hanada, H. Lewis, Stability of the future of LEO environment, in: Inter-Agency Space Debris Coordination Committee, vol, 8, Jan 2013, IADC-12-08, Rev. 1.

[2] Donald J. Kessler, Burton G. Cour-Palais, Collision frequency of artificial satellites: the creation of a debris belt, J. Geophys. Res, Space Phys. 83 (A6) (1978) 2637-2646.

[3] Joseph A. Carroll, Tether applications in space transportation, Acta Astronaut. 13 (4) (1986) 165-174.

[4] V.V. Beletsky, EM. Levin, Dynamics of Space Tether Systems, American Astronautical Society. Advances in the Astronautical Science, vol. 83, 1993.

[5] M.L Cosmos, E.C. Lorenzini (Eds.), Tethers in Space Handbook, Smithsonian Astrophysical Observatory, 1997.

[6] M.P. Cartmell, D.J. McKenzie, A review of space tether research, Prog. Aerosp. Sci. 44 (1) (2008) 1-21,

[7] V. Aslanov, A.S. Ledkov, Dynamics of Tethered Satellite Systems, Elsevier, 2017.

[8] G. Sánchez-Arriaga, J.R. Sanmartín, E.C. Lorenzini, Comparison of technologies for deorbiting spacecraft from low-Earth-orbit at end of mission, in: The Fifth International Conference on Tethers in Space, Acta Astronaut. 138 (2017) $536-542$

[9] Carmen Pardini, Toshiya Hanada, Paula H. Krisko, Benefits and risks of using electrodynamic tethers to de-orbit spacecraft, in: 57th International Astronautical Congress, Valencia, Spain, Oct 02-06, 2006, Acta Astronaut, 64 (5-6) (MARAPR 2009) 571-588.

[10] S.B. Khan, J.R. Sanmartin, Survival probability of round and tape tethers against debris impact, J. Spacecr. Rockets 50 (May 2013) 603-608.

[11] Shaker Bayajid Khan, A. Francesconi, C. Giacomuzzo, E.C. Lorenzini, Survivability to orbital debris of tape tethers for end-of-life spacecraft de-orbiting, Aerosp. Sci. Technol. 52 (MAY 2016) 167-172.

[12] J.R. Sanmartin, A. Sanchez Torres, S.B. Khan, G, Sanchez-Arriaga, M. Charro, Optimum sizing of bare-tape tethers for de-orbiting satellites at end of mission, Adv. Space Res. 56 (10 2015) 1485-1492.

[13] ]. Pelaez, E,C, Lorenzini, O. Lopez-Rebollal, M. Ruiz, A new kind of dynamic instability in electrodynamic tethers, in: AAS/AIAA 10th Space Flight Mechanics Meeting, Clearwater, Florida, Jan 23-26, 2000, J. Astronaut. Sci. 48 (4) (Oct-Dec 2000) $449-476$

[14] K.D. Kumar, Review of dynamics and control of nonelectrodynamic tethered satellite systems, J. Spacecr. Rockets 43 (4) (JUL-AUG 2006) 705-720.

[15] A.K. Misra, Dynamics and control of tethered satellite systems, in: 54th International-Astronautical-Federation Congress, IAF, Bremen, Germany, Sep 29-Oct 03, 2003, Acta Astronaut. 63 (11-12) (DEC 2008) 1169-1177.

[16] Z.H. Zhu, Mission design of deorbiting spacecraft using electrodynamic tethers (descent), in: The International Conference on Aerospace System Science and Engineering, ICASSE 2017, Shanghai, China, 2017.

[17] Panfeng Huang, Yakun Zhao, Fan Zhang, Jun Ma, Zhongjie Meng, Zhengxiong Liu, Yizhai Zhang. Deployment/retraction of the rotating Hub-Spoke Tethered Formation System, Aerosp. Sci. Technol. 69 (Oct 2017) 495-503.

[18] Xin Sun, Rui Zhong, Switched propulsion force libration control for the low thrust space tug system, Aerosp, Sci. Technol. 80 (2018) 281-287.
[19] Vladimir S. Aslanov, Alexander S. Ledkov, Swing principle in tether-assisted return mission from an elliptical orbit, Aerosp. Sci. Technol. 71 (2017) 156-162.

[20] J.R. Sanmartin, M. Martinez-Sanchez, E. Ahedo, Bare wire anodes for electrody namic tethers, J. Propuls. Power 9 (1993) 353-360.

[21] J.D. Williams, J.R. Sanmartín, L.P. Rand, Low work-function coating for an en tirely propellantless bare electrodynamic tether, IEEE Trans. Plasma Sci. 40 (May 2012) 1441-1445.

[22] G. Sanchez-Arriaga, Xin Chen, Modeling and performance of electrodynamic low-work-function tethers with photoemission effects, J. Propuls. Power 34 (1) (JAN 2018) 213-220.

[23] C. Bombardelli, G. Sanchez-Arriaga, System electric power generation in orbit using floating conductor cables, patent es2562713b2, 01 2017. Spanish Patent ES2562713B2.

[24] G. Sanchez-Arriaga, C. Bombardelli, Orbit propulsion system by floating con ductor cables, 05 2017. Spanish Patent WO2017081351A1

[25] G. Sánchez-Arriaga, X. Chen, E.C. Lorenzini, Optimal design and deorbiting performance of thermionic bare tethers in geostationary transfer orbits, J. Propuls. Power 33 (2) (2017) 425-432.

[26] J.R. Sanmartin, R.D. Estes, E.C. Lorenzini, S.A. Elaskar, Efficiency of electrody namic tether thrusters, J. Spacecr. Rockets 43 (3) (May 2006) 659-666.

[27] G.V. Khazanov, E.N. Krivorutsky, D.L. Gallagher, Electrodynamic bare tether systems as a thruster for the momentum-exchange/electrodynamic reboost (MXER) project, J. Geophys. Res. Space Phys. 111 (A4) (2006).

[28] L. Johnson, M. Hermann, International space station electrodynamic tether reboost, in: Space Technology and Applications International Forum, 1999.

[29] Irwin E. Vas, Thomas J. Kelly, Ethan A. Scarl, Space station reboost with elec trodynamic tethers, J. Spacecr. Rockets 37 (2) (Mar 2000) 154-164.

[30] Joseph Bonometti, Kirk Sorensen, Ralph Jansen, John Dankanich, Kyle Frame Free re-boost electrodynamic tether for the international space station, in: Joint Propulsion Conferences, American Institute of Aeronautics and Astronautics, Jul 2005, AIAA-2005-4545, https://doi,org/10.2514/6.2005-4545.

[31] J.G. Laframboise, L.W. Parker, Probe design for orbit-limited current collection. Phys. Fluids 16 (1973) 629-636.

[32] O.W. Richardson, The Emission of Electricity from Hot Bodies, Longmans, Green, and Co., 1916.

[33] J.R. Sanmartín, Xin Chen, G. Sánchez-Arriaga, Analysis of thermionic bare tether operation regimes in passive mode, Phys. Plasmas 24 (1) (2017) 013515.

[34] Xin Chen, J.R. Sanmartín, Low work-function thermionic emission and orbitalmotion-limited ion collection at bare-tether cathodic contact, Phys. Plasmas 22 (5) (2015)

[35] E. Choiniere, Theory and Experimental Evaluation of a Consistent Steady-State Kinetic Model for Two-Dimensional Conductive Structures in Ionospheric Plasmas with Application to Bare Electrodynamic Tethers in Space, PhD thesis, University of Michigan, 2004.

[36] J.R. Sanmartin, E. Choiniere, B.E. Gilchrist, J.-B. Ferry, M. Martinez-Sanchez, Bare-tether sheath and current: comparison of asymptotic theory and kinetic simulations in stationary plasma, IEEE Trans. Plasma Sci. 36 (October 2008) 2851-2858.

[37] B.S. Yu, P.B. Dai, D.P. Jin, Modeling and dynamics of a bare tape-shaped tethered satellite system, Aerosp. Sci. Technol. 79 (2018) 288-296.

[38] M, Sanjurjo, G. Sanchez-Arriaga, J. Pelaez, Efficient computation of current col lection in bare electrodynamic tethers in and beyond OML regime, J. Aerosp. Eng. 0 (2014) 04014144. 